\title{
Free radical scavenging reverses fructose-induced salt-sensitive hypertension
}

This article was published in the following Dove Press journal: Integrated Blood Pressure Control

\section{Zachary P Zenner \\ Kevin L Gordish \\ William H Beierwaltes \\ Department of Internal Medicine, Hypertension and Vascular Research Division, Henry Ford Hospital, Detroit, MI, USA}

Correspondence: William H Beierwaltes Department of Internal Medicine, Hypertension and Vascular Research Division, Henry Ford Hospital, 7088 E\&R Building, 2799 W. Grand Boulevard, Detroit, MI 48202, USA

$\mathrm{Tel}+\mathrm{I} 3139167494$

Fax + I 3139165284

Email patandwill@msn.com

\begin{abstract}
We have previously reported that a moderate dietary supplementation of $20 \%$ fructose but not glucose leads to a salt-sensitive hypertension related to increased proximal sodiumhydrogen exchanger activity and increased renal sodium retention. We also found that while high salt increased renal nitric oxide formation, this was retarded in the presence of fructose intake. We hypothesized that at least part of the pathway leading to fructose-induced salt-sensitive hypertension could be due to fructose-induced formation of reactive oxygen species and inappropriate stimulation of renin secretion, all of which would contribute to an increase in blood pressure. We found that both $20 \%$ fructose intake and a high-salt diet stimulated 8 -isoprostane excretion. The superoxide dismutase (SOD) mimetic tempol significantly reduced this elevated excretion. Next, we placed rats on a high-salt diet (4\%) for 1 week in combination with normal rat chow or $20 \%$ fructose with or without chronic tempol administration. A fructose plus highsalt diet induced a rapid increase $(15 \mathrm{mmHg})$ in systolic blood pressure and reversed high salt suppression of plasma renin activity. Tempol treatment reversed the pressor response and restored high salt suppression of renin. We conclude that fructose-induced salt-sensitive hypertension is driven by increased renal reactive oxygen species formation associated with salt retention and an enhanced renin-angiotensin system.
\end{abstract}

Keywords: reactive oxygen species, tempol, sodium, renin, oxidative stress

\section{Introduction}

Rats fed a high-fructose diet have been characterized as a model of insulin resistance and associated hypertension. ${ }^{1,2}$ An animal model was first reported by Hwang et $\mathrm{al}^{3}$ in rats fed with very high fructose ( $66 \%$ of their caloric intake) which developed type 2 diabetes, hyperinsulinemia, hypertriglyceridemia, metabolic syndrome, and hypertension within 2 weeks. Similar studies using glucose had no such effect. However, this model has been criticized because of the excessive amount of fructose required to produce these results. When similar studies have been carried out using just $20 \%$ fructose in the drinking water but without high salt (HS), it took much longer (8-12 weeks) to develop systolic hypertension, and this was accompanied by parameters such as obesity, hypertrophy of adipocytes, hypertriglyceridemia, and hyperglycemia. ${ }^{4}$

It has been suggested that fructose-induced hypertension results from excess renal salt retention. ${ }^{5}$ Our laboratories have previously shown that fructose, at levels consistent with those consumed by the upper 20th percentile of the human population, does not induce hypertension in rats during 2 weeks if consuming a normal salt intake $(0.4 \%$ $\mathrm{NaCl})$. However, combining fructose and an $\mathrm{HS}$ intake $(4.0 \% \mathrm{NaCl})$ resulted in a 
rapid and significant rise in blood pressure (15-20 $\mathrm{mmHg}$ ) within 1 week. ${ }^{6,7}$ We also reported that combining HS diet with $20 \%$ fructose (but not with glucose) intake led to significant sodium retention associated with the increase in blood pressure. ${ }^{7}$ Further, we reported ${ }^{6}$ that fructose but not glucose acutely stimulated luminal sodium-hydrogen exchanger activity in the proximal tubule. These studies led us to conclude that $20 \%$ fructose intake results in a predisposition for salt-sensitive hypertension well before the previously reported ${ }^{4}$ effects of a $20 \%$ fructose diet alone on blood pressure over 8-12 weeks.

The renin-angiotensin system is an important regulator of blood pressure. ${ }^{89}$ Inappropriate elevation of renin can cause both salt-sensitive and salt-independent hypertension. Renin activity is the rate-limiting step in the formation of angiotensin II (Ang II). Salt sensitivity of blood pressure in renin-dependent hypertension results largely from the effects of Ang II on the kidney primarily due to increases in sodium reabsorption by the nephron. Ang II increases activation and production of proinflammatory cytokines in the kidney, which are believed to play an important role in the development of salt-sensitive hypertension. ${ }^{10-12}$ Ang II induces the production of reactive oxygen species in the proximal tubule mainly through the activation of nicotinamide adenine dinucleotide phosphate (NADPH) oxidase, leading to tubulointerstitial injury. ${ }^{13}$ These products also mediate various signaling pathways involved in intrarenal inflammation and hypertension. Several studies have implicated activation of renin secretion by a high-fructose diet. ${ }^{14,15}$

We found ${ }^{7}$ that fructose acutely enhances the sensitivity of angiotensin-stimulated sodium-hydrogen exchanger activity in the rat proximal tubule. Queiroz-Leite et $\mathrm{al}^{16}$ have similarly found that fructose stimulates proximal sodium-hydrogen exchanger 3 (NHE3) activity. So, while Ang II may directly affect sodium reabsorption in this model, it is not known whether moderate fructose intake directly stimulates renin secretion or if it interacts with Ang II and an HS diet to cause hypertension, or a combination of both.

Reactive oxygen species such as superoxide $\left(\mathrm{O}_{2}^{-}\right)$and hydrogen peroxide $\left(\mathrm{H}_{2} \mathrm{O}_{2}\right)$ are increased in the renal cortex in genetic models of salt-sensitive hypertension. ${ }^{17} \mathrm{HS}$ diet has also been shown to increase 8-isoprostane excretion, a marker for increased renal reactive oxygen species formation. ${ }^{18}$ Besides having intrinsic vasoconstrictor properties and decreasing the bioavailability of the intrinsic vasodilator and natriuretic nitric oxide, ${ }^{19,20}$ it has been reported that reactive oxygen species can stimulate the secretion of renin ${ }^{21}$ directly from the juxtaglomerular cells. Further, Ang II is a well-known stimulator of reactive oxygen species. ${ }^{18}$ High fructose has also been linked to increased reactive oxygen species production. In vitro, fructose added to the culture media of skeletal muscle cell $\mathrm{s}^{22}$ provoked mitochondrial reactive oxygen species formation, mitochondrial dysfunction and progressive apoptosis. In vivo, fructose ingestion prior to aerobic exercise in male athletes resulted in a significant rise in oxidative stress, ${ }^{23}$ not seen with glucose ingestion. From all of these related observations, we hypothesized that at least part of the pathway leading to fructose-induced salt-sensitive hypertension could be due to fructose-induced increased reactive oxygen species and inappropriate stimulation of renin secretion, all of which would contribute to an increase in blood pressure.

\section{Materials and methods}

Our studies employed male Sprague Dawley rats initially weighing 220-300 g (8-10 weeks of age), which were obtained from Charles Rivers Laboratories (Kingston, NY, USA). All procedures were approved by the Henry Ford Health System Institutional Animal Care and Use Committee (IACUC) and adhered to the guiding principles in the care and use of experimental animals in accordance with the National Institute of Health (NIH) guidelines. Henry Ford Hospital operates an American Association for Laboratory Animal Care (AALAC)-certified animal care facility.

\section{Protocol I: urinary 8-isoprostane excretion with fructose and HS rats Groups}

This study was gleaned from data obtained over 2 years using identical protocols of 2-week duration, including all rats from protocol 2. After the initial training period, rats were placed on one of six diet permutations including 1) a control (C) diet, eating standard rat chow containing $0.4 \% \mathrm{NaCl}$ (Harlan Teklad, Madison, WI, USA) over 2 weeks $(\mathrm{n}=9), 2)$ an HS diet, in which the standard diet was replaced by an HS diet $(4 \% \mathrm{NaCl}$; Harlan Teklad) during the second week of the protocol $(n=17), 3)$ an HS plus tempol (HS + T) treatment, where the rats received the free radical scavenger tempol (Sigma-Aldrich Co., St Louis, MO, USA) in the drinking water ( $15 \mathrm{mg}$ tempol/400 $\mathrm{g}$ rat/day) throughout the 2 weeks and were switched to the HS diet during the second week $(n=9), 4)$ fed with fructose (F), in which the rats received the normal rat chow, but their drinking water was supplemented with $20 \%$ fructose (Sigma-Aldrich Co.) throughout the 2-week study period $(\mathrm{n}=9), 5)$ HS plus fructose (HS $+\mathrm{F}$ ), in which the rats received the $20 \%$ fructose drinking water throughout the 2 weeks and were switched to the HS 
diet during the second week of the study $(n=27)$, and 6) HS plus fructose plus tempol $(\mathrm{HS}+\mathrm{F}+\mathrm{T})$ treatment, in which the rats received both $20 \%$ fructose plus tempol in their drinking water throughout the 2 weeks and were switched to the HS diet during the second week $(n=7)$. During the final $48 \mathrm{~h}$ of the study period, the rats were moved to metabolic caging and allowed to acclimate to the new environment for 1 day. For the second $24 \mathrm{~h}$, urine was collected and the volume was measured gravimetrically.

\section{Urinary 8-isoprostane excretion}

A portion of each urine sample was assayed for urinary excretion of 8-isoprostane, a marker of oxidative stress. ${ }^{24}$ Urine collection vials contained $200 \mu \mathrm{L}$ of anti-anti, an antifungal, antimicrobial agent (Thermo Fisher Scientific, Waltham, MA, USA). Collected urine samples were centrifuged to eliminate particulate contamination. An 8-isoprostane enzyme-linked immunosorbent assay (ELISA) kit was used to measure urinary concentration (Cayman Chemical, Ann Arbor, MI, USA). Urinary concentrations were multiplied by urine volumes to obtain $24 \mathrm{~h}$ 8-isoprostane excretion values, expressed as ng/24 h.

\section{Protocol 2: systolic blood pressure with tempol treatment of fructose- and HS-fed rats}

Male Sprague Dawley rats weighing 226-251 g were housed in standard caging. Prior to the beginning of the protocol, rats were allowed free access to distilled water and normal rat chow containing $0.4 \%$ sodium chloride (Harlan Teklad). In addition, rats were pretrained over 7-10 days on a noninvasive tail cuff plethysmography multichannel system (Kent Scientific, Torrington, CT, USA). When the protocol commenced, systolic blood pressure was measured every other day for the duration of the 2-week protocol. In our experience, with proper training and consistent methodology, tail cuff systolic blood pressure measurements are reliable and match systolic blood pressure data obtained using telemetry.

Rats were distributed into one of four treatment groups. These included 1) the HS diet control group (HS-C), in which the standard diet was replaced by an $\mathrm{HS}$ diet $(4 \% \mathrm{NaCl})$ during the second week of the protocol $(n=8) ; 2)$ the HS + T-treated group, where the rats received the free radical scavenger tempol (Sigma-Aldrich Co.) in the drinking water (15 mg tempol/400 $\mathrm{g}$ rat/day) throughout the 2 weeks and were switched to the HS diet during the second week $(n=9)$; 3) the HS + F group, in which the rats received drinking water containing $20 \%$ fructose in their drinking water throughout the 2 weeks and were switched to the HS diet during the second week of the study $(\mathrm{n}=9)$; and 4$)$ the $\mathrm{HS}+\mathrm{F}+\mathrm{T}$ group, in which the rats received both $20 \%$ fructose plus tempol in their drinking water throughout the 2 weeks and were switched to the HS diet during the second week $(n=8)$. We did not include any groups on a normal salt diet as we have previously documented their responses extensively in comprehensive metabolic balance studies ${ }^{7}$ and blood pressure did not change within this 2 -week time frame.

\section{Diet and body weight}

During the first week of the 14-day protocol, all rats were fed normal rat chow $(0.4 \% \mathrm{NaCl})$. For the second week, the diet in every group was switched to $\mathrm{HS}(4.0 \% \mathrm{NaCl})$. Both the normal rat chow and the HS diets were obtained from Harlan Teklad, and otherwise were identical in nutrient and caloric content. The rat chow had an energy density of $3 \mathrm{kcal} / \mathrm{g}$ (Harlan Data Sheet \#8640). The energy density of fructose is $4 \mathrm{kcal} / \mathrm{g}$.

Body weight was recorded every other day using a covered rodent triple-beam balance (Ohaus, Parsippany, NJ, USA).

\section{Drinking water/tempol + fructose administration}

Prior to the 2-week protocol, an initial training period of 1 week was run. Rats were then assigned to one of four dietary treatment groups. To assure consistent drug intake, all rats were given a volume of $35 \mathrm{~mL}$ of their assigned drinking water once every $24 \mathrm{~h}$ for week 1 of the protocol. When scheduling allowed, rats of all groups were given free access to distilled water but only after drinking their initial treatment volume. For the second week of the protocol, assigned drinking water doses were increased in volume from $35 \mathrm{~mL}$ to $45 \mathrm{~mL}$. This change was designed to accommodate an anticipated greater thirst with the change to an HS diet in the second week of the protocol. Each group's drinking water solution was prepared every 2-3 days apart and stored in a refrigerator to limit microbial growth.

\section{Sodium excretion}

Two $24 \mathrm{~h}$ urine collections were obtained over the final $48 \mathrm{~h}$ of the 2-week protocol. The rats were placed in metabolic cages. In these, their urine drained freely through the wire mesh bottom of the cage, then through a wire mesh feces trap, and into a $50 \mathrm{~mL}$ conical tube containing $200 \mu \mathrm{L}$ of antibiotic-antimycotic solution to prevent growth of bacteria (Thermo Fisher Scientific). All the initial $24 \mathrm{~h}$ urine collection samples were discarded, and only the urines from the final $24 \mathrm{~h}$ of the protocol were analyzed. The volume of these final $24 \mathrm{~h}$ urine samples was measured by weight using an Ohaus electronic balance (Mettler Toledo, Columbus, OH, USA). 
Urine samples were spun at $3500 \mathrm{rpm}$ at $4^{\circ} \mathrm{C}$ for $10 \mathrm{~min}$ to remove sediment. Samples were transferred to Eppendorf tubes and placed in a $-80^{\circ}$ freezer. Sodium excretion was calculated from $24 \mathrm{~h}$ urine volumes and sodium concentrations measured by a Nova Biomedical 1 electrolyte analyzer (Nova Biomedical, Waltham, MA, USA).

\section{Plasma renin activity (PRA)}

At the end of the protocol, rats were euthanized via decapitation using a rat guillotine (Harvard Bioscience, Cambridge, MA, USA). Promptly after decapitation, the first $3 \mathrm{~s}$ of trunk blood were collected for PRA analyses in $15 \mathrm{~mL}$ tubes containing $200 \mu \mathrm{L}$ of $6 \%$ EDTA in $0.9 \% \mathrm{NaCl}$. This collection method has been approved by our IACUC as it avoids the abnormal stimulation of PRA by the renal baroreceptor reflex and by anesthesia, which would otherwise abnormally increase PRA. ${ }^{25}$ Additional blood samples were taken using either $200 \mu \mathrm{L}$ of $6 \%$ EDTA (Sigma-Aldrich Co.), or $100 \mu \mathrm{L}$ of sodium heparin (Sagent Pharmaceuticals, Schaumburg, IL, USA) as anticoagulants. Blood samples were spun at $1,000 \times g$ at $4^{\circ} \mathrm{C}$ for $10 \mathrm{~min}$, and the plasma was separated and stored at $-80^{\circ} \mathrm{C}$ until further analysis.

PRA was analyzed by generation of angiotensin I (Ang I; ng Ang I $\times \mathrm{mL}^{-1} \times \mathrm{h}^{-1} \times \mathrm{min}^{-1}$ ) using a Gamma Coat RIA kit (DiaSorin, Stillwater, MN, USA) as previously described ${ }^{26}$ and according to the manufacturer's instructions. Following blood collection, the kidneys were excised, decapsulated, blotted, weighed, split, and inspected for gross abnormalities (hydronephrosis, lobular appearance, ischemic areas, etc), and none were found. In previous studies characterizing this model, within this 2-week protocol duration, we did not observe any microscopic histological changes nor proteinuria.

\section{Blood glucose}

Blood glucose was measured at the end of the protocol. For $24 \mathrm{~h}$ prior to euthanasia, the rat groups were fasted, but allowed access to water. Following euthanasia, blood droplets were collected from trunk blood to measure fasting plasma glucose levels. Blood glucose was measured with a True Result Blood Glucose Monitor (CVS, Woonsocket, RI, USA).

\section{Statistical treatments}

Single comparisons were run using a Student's $t$-test. Change between two periods was evaluated using a paired $t$-test. Multiple comparisons were run using analysis of variance (ANOVA) or ANOVA with repeated measures. For a post hoc test, we used the Newman-Keuls test. Statistical significance was established by a $p$-value of at least 0.05 .

\section{Results}

\section{Protocol I: urinary 8-isoprostane excretion with fructose and HS rats}

To test the effect of $20 \%$ fructose and an HS diet on renal oxidative stress, we measured $24 \mathrm{~h}$ urinary 8 -isoprostane excretion. 8-Isoprostane excretion in the six study groups (Figure 1) were: 1) (C) $16.38 \pm 1.30,2$ ) (HS) $26.69 \pm 0.51$, 3) $(\mathrm{HS}+\mathrm{T}) 26.86 \pm 4.60$, 4) $(\mathrm{F}) 32.18 \pm 3.895)(\mathrm{HS}+\mathrm{F})$ $29.84 \pm 1.96$, and 6) $(\mathrm{HS}+\mathrm{F}+\mathrm{T}) 20.80 \pm 3.40 \mathrm{ng} / 24 \mathrm{~h}$. By the end of the 2-week study period, 8-isoprostane was significantly higher by $80-90 \%(p<0.01)$ in all groups fed either HS, $20 \%$ fructose, or a combination of the two (Figure 1) compared to controls. There were no differences among the different HS or fructose-fed groups with elevated 8 -isoprostane excretion. Tempol treatment did not affect the increased 8-isoprostane excretion in the HS-only group, but significantly reduced 8-isoprostane excretion in the HS + F + T group by $40 \%(p<0.028$; Figure 1$)$. Thus, both HS and $20 \%$ fructose increased this marker for oxidative stress, but when combined the effects were not additive. Tempol treatment reduced this elevation of 8-isoprostane only when both HS and fructose were present.

\section{Protocol 2: systolic blood pressure with tempol treatment of fructose and HS rats Diet and body weight}

Body weights between the four groups were similar both at the beginning and at the end of the 2-week protocol (Table 1). Thus, growth rate was also similar among the four groups (Table 1). While growth in the two groups treated with tempol

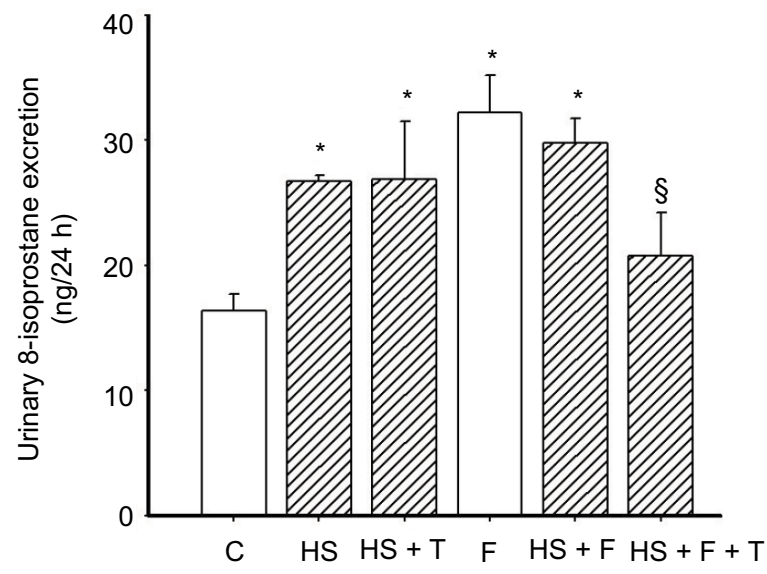

Figure I Protocol I: 8-isoprostane excretion in the six different groups. Notes: $\mathrm{C}=$ control, normal salt $(0.4 \% \mathrm{NaCl})$ diet; $\mathrm{HS}(4.0 \% \mathrm{NaCl})$ diet; $\mathrm{HS}+\mathrm{T}, \mathrm{HS}$ plus tempol-treated; $\mathrm{F}=20 \%$ fructose-fed group; $\mathrm{HS}+\mathrm{F}, 20 \%$ fructose-fed plus an $\mathrm{HS}$ diet; HS + F + T, 20\% fructose-fed plus an HS diet and treated with tempol. ${ }^{*} p<0.01$ vs $C$ (adjusted for multiple comparisons), ${ }^{s} p<0.028$ vs $\mathrm{HS}+$ fructose-fed group. Abbreviations: C, control; F, fructose; HS, high salt; T, tempol. 
Table I Protocol 2: body weight and kidney weight

\begin{tabular}{|c|c|c|c|c|}
\hline \multirow[t]{2}{*}{ Parameter } & \multicolumn{4}{|l|}{ Diet group } \\
\hline & HS-C & HS + T & HS + F & $H S+F+T$ \\
\hline Body weight at day “0” (g) & $260 \pm 10$ & $259 \pm 8$ & $258 \pm 6$ & $254 \pm 6$ \\
\hline Body weight at 2 weeks (g) & $321 \pm 10$ & $311 \pm 6$ & $321 \pm 6$ & $304 \pm 4$ \\
\hline Growth rate (g/day) & $4.3 \pm 0.5$ & $3.7 \pm 0.4$ & $4.5 \pm 0.5$ & $3.9 \pm 0.4$ \\
\hline Left kidney weight (g) & $1.19 \pm 0.05$ & $1.15 \pm 0.03$ & $1.11 \pm 0.04$ & $I .11 \pm 0.03$ \\
\hline
\end{tabular}

Abbreviations: $\mathrm{C}$, control; $\mathrm{F}$, fructose; $\mathrm{HS}$, high salt; $\mathrm{T}$, tempol.

appeared somewhat slower, none of these differences were statistically significant. Kidney weights at the end of the study period were also similar among the four groups.

\section{Systolic blood pressure}

The mean systolic blood pressures within the four groups $(138 \pm 2 \mathrm{mmHg})$ were similar at the beginning of the protocol after the training period and were not still not different from each other after a week on the high-fructose diet (Figure 2A): the HS-C group, $144 \pm 6 \mathrm{mmHg}$; the HS + T-treated group, $142 \pm 4 \mathrm{mmHg}$; the HS + F group, $142 \pm 4 \mathrm{mmHg}$; the HS + F + T-treated group, $132 \pm 12 \mathrm{mmHg}$. Over the final week, when HS was added to the diets, the blood pressure in the HS $+\mathrm{F}$ group began increasing within 4 days (150 \pm $5 \mathrm{mmHg}, p<0.05$ ) and increased even further (Figure 2A) by the end of the protocol. After a week of receiving both $20 \%$ fructose and HS, the blood pressure was increased by over $15 \mathrm{mmHg}(156 \pm 6 \mathrm{mmHg}, p<0.01)$; a rise similar to what we have previously reported. ${ }^{7}$ Blood pressure in the other three groups remained unchanged after 7 days on HS: HS-C group, $139 \pm 4 \mathrm{mmHg}$; HS + T-treated group, $138 \pm$ $3 \mathrm{mmHg}$; HS + F + T-treated group, $136 \pm 3 \mathrm{mmHg}$. Thus, tempol treatment completely reversed the increase in blood pressure seen with the combination of $20 \%$ fructose and HS (Figure 2B).

\section{Drinking water/tempol + fructose administration}

\section{Sodium and water excretion}

The $24 \mathrm{~h}$ sodium excretion in the four groups on the last day of the 2-week protocols was not statistically different from each other. The values were 1) HS-C, $2.20 \pm 0.65 \mathrm{mM} / 24 \mathrm{~h}$; 2) $\mathrm{HS}+\mathrm{T}$, 3) $1.63 \pm 0.25 \mathrm{mM} / 24 \mathrm{~h}$; 4) $\mathrm{HS}+\mathrm{F}, 1.74 \pm$ $0.27 \mathrm{mM} / 24 \mathrm{~h}$, and HS + F + T, $2.14 \pm 0.24 \mathrm{mM} / 24 \mathrm{~h}$.

The $24 \mathrm{~h}$ urinary excretion in the four groups on the last day of the 2-week protocols were 1) HS-C, $27.3 \pm$ $3.9 \mathrm{~mL} / 24 \mathrm{~h}$; 2) HS + T, $12.0 \pm 2.7 \mathrm{~mL} / 24 \mathrm{~h}$; 3) HS + F, 25.7 $\pm 1.1 \mathrm{~mL} / 24 \mathrm{~h}$, and 4) $\mathrm{HS}+\mathrm{F}+\mathrm{T}, 21.1 \pm 2.7 \mathrm{~mL} / 24 \mathrm{~h}$. The volume output was similar in all groups except the HS $+\mathrm{T}$

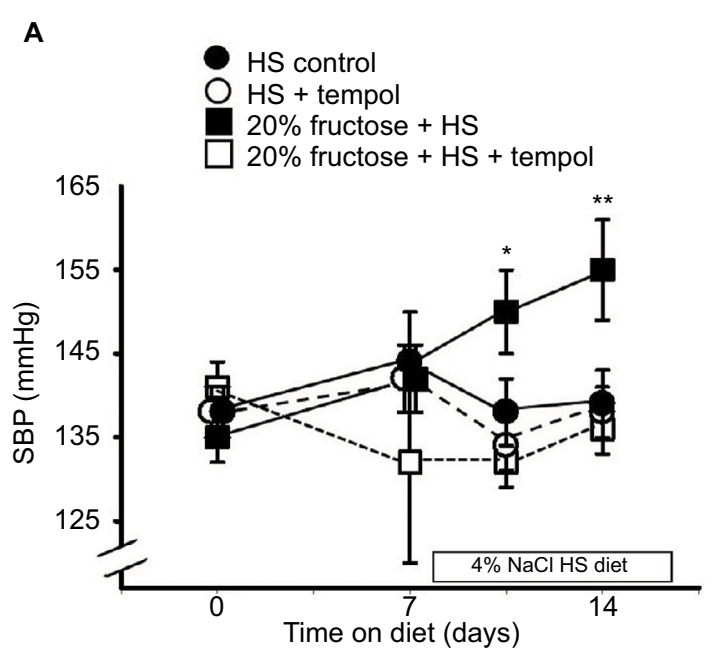

B

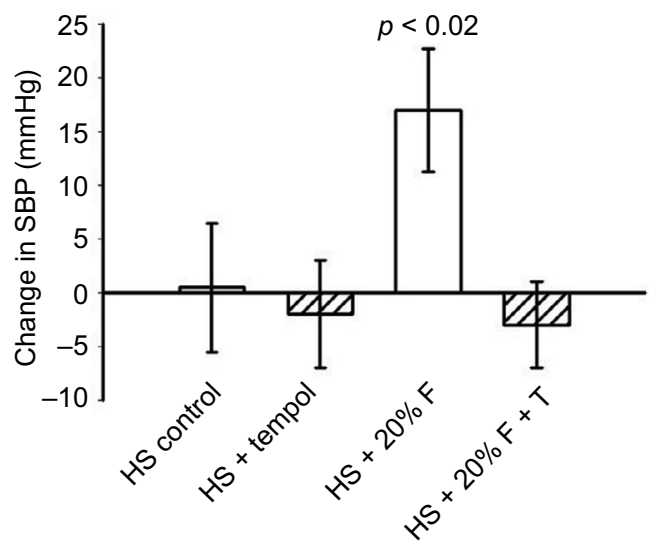

Figure 2 Effect of the different diets/treatments on systolic blood pressure. Notes: Protocol 2: (A) the SBP over 2 weeks on $20 \%$ fructose with HS diet during the second week and the effects of tempol treatment. ${ }^{*} p<0.05$, ** $p<0.01$ vs control SBP at day "0." (B) The changes in SBP over the 2 weeks of the study in each group.

Abbreviations: $F$, fructose; $\mathrm{HS}$, high salt; SBP, systolic blood pressure; $T$, tempol.

group, which had a volume only half that of the other groups $(p<0.02)$. We found that the rats did not like tempol in their water, which led to reduced intake and greater variability in the HS $+\mathrm{T}$ group. However, when fructose was added (in the HS + F + T group), the sweetness overcame this distaste and water intake was normalized. 


\section{PRA}

PRA in the HS-C group was $2.28 \pm 0.53 \mathrm{ng}$ Ang I/mL/h. The HS + T group was similar at $1.84 \pm 0.27 \mathrm{ng}$ Ang $\mathrm{I} / \mathrm{mL} / \mathrm{h}$. The addition of $20 \%$ fructose combined with HS tended to increase PRA to $3.57 \pm 0.52 \mathrm{ng}$ Ang I/mL/h (Figure 3) as we have reported previously, ${ }^{7}$ this increase did not reach a statistically significant difference from HS-C. However, the combination of $20 \%$ fructose plus HS treated with tempol decreased PRA by 50\% compared to the same treatment without tempol $(p<0.025)$ to a value of $1.79 \pm 0.37 \mathrm{ng}$ Ang $\mathrm{I} / \mathrm{mL} / \mathrm{h}$, and this value was similar to HS-C with or without tempol (Figure 3).

\section{Blood glucose}

Fasting blood glucose levels at the end of the protocol were not significantly different between the four groups: HS-C, $52 \pm 2 \mathrm{mg} / \mathrm{dL} ; \mathrm{HS}+\mathrm{T}, 53 \pm 2 \mathrm{mg} / \mathrm{dL} ; \mathrm{HS}+\mathrm{F}, 58 \pm 2 \mathrm{mg} / \mathrm{dL}$; and $\mathrm{HS}+\mathrm{F}+\mathrm{T}, 57 \pm 2 \mathrm{mg} / \mathrm{dL}$.

\section{Discussion}

We have previously reported ${ }^{6,7}$ that a diet supplemented with $20 \%$ fructose in the drinking water of rats over a duration of just 2 weeks, when combined with an HS diet, can induce salt-sensitive hypertension prior to the onset of other metabolic alterations. This was coincident with increased renal proximal NHE3 activity and enhanced sensitivity to Ang II in isolated proximal tubules, ${ }^{6}$ as well as increased sodium retention, decreased renal nitric oxide, ${ }^{7}$ and diminished suppression of PRA with an HS diet. ${ }^{7}$ In the current study, we again found that the combination of $20 \%$ fructose and an HS diet led to significant increases in systolic blood pressure in only 1 week. Likewise, HS suppression of PRA was blunted

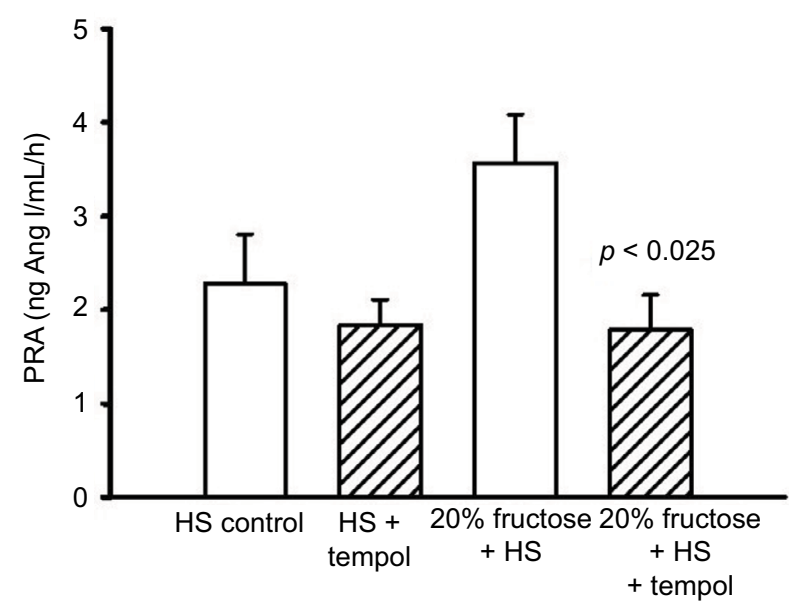

Figure 3 Protocol 2: PRA at the end of the experimental protocol. Note: $p$-value vs similar diet without tempol treatment. Abbreviations: Ang I, angiotensin I; HS, high salt; PRA, plasma renin activity. by fructose intake. We also now report that both an HS diet and $20 \%$ fructose intake increase urinary 8 -isoprostane excretion suggesting each lead to an increase in renal reactive oxygen species formation. Because of this, we tested if the oxygen-free radical scavenging, superoxide dismutase (SOD) mimetic tempol, would affect the increase in blood pressure seen with a combination of these two dietary challenges of reactive oxygen species. We hypothesized that at least part of the pathway leading to fructose-induced salt-sensitive hypertension could be due to fructose-induced formation of reactive oxygen species and inappropriate stimulation of renin secretion, all of which would contribute to an increase in blood pressure. What we found was a rapid and dramatic increase in systolic blood pressure in response to the combination of $20 \%$ fructose and HS, similar to what we have previously reported, ${ }^{6,7}$ and further that this was completely reversed in rats chronically treated with tempol. Further, while we found (as before ${ }^{7}$ ) that fructose blunted the HS suppression of PRA, but now we found that this effect on PRA was significantly reduced (reversed) with the addition of tempol. Our data suggest that the factors leading to fructoseinduced, salt-sensitive hypertension may be in part driven by increased renal oxygen free radical formation.

HS diet is known to increase renal cortical reactive oxygen production, ${ }^{27,28}$ as well as cortical nitric oxide synthesis. ${ }^{29}$ We and others have previously reported ${ }^{7,28}$ an important interaction between nitric oxide and superoxide in maintaining both renal blood flow and sodium excretion in normotensive rats. Nitric oxide is known to be an important natriuretic factor in the proximal tubule. ${ }^{30} \mathrm{We}$ have also reported that $\mathrm{HS}$ intake dobles the $\mathrm{NO}_{2} / \mathrm{NO}_{3}$ excretion (as a marker of renal NO production), presumably because of its natriuretic properties,${ }^{30}$ but in the presence of $20 \%$ fructose this response is significantly attenuated. This suggests that the fructose intake either directly or indirectly diminishes nitric oxide bioavailability.

It is well established that renal production of reactive oxygen species, primarily by NADPH oxidase in the kidney, is an important factor in regulating renal function, ${ }^{27}$ and excesses in its activity could lead to renal dysfunction and hypertension. As little as $10 \%$ fructose in the drinking water of rats leads to systemic oxidative stress and related pro-inflammatory conditions. ${ }^{31}$

Tempol is a cell-permeable SOD mimetic which scavenges free radicals and attenuates superoxide anion and peroxynitrite-induced inflammation. ${ }^{32}$ It is water soluble and can be easily administered in the drinking water. ${ }^{33}$ Tempol is a redox-cycling nitroxide which metabolizes many reactive 
oxygen species. ${ }^{34}$ Thus, it does not have selectivity so that the specific reactive oxygen species generated cannot be determined from these results. Tempol has been reported to have antihypertensive properties in various rat models of hypertension. ${ }^{27}$ In the spontaneously hypertensive rat, Yanes et a ${ }^{33}$ found that 2 weeks of tempol treatment reduced oxidative stress and reduced mean arterial pressure. This response was found to be nitric oxide dependent but independent of the renin-angiotensin system. In $2 \mathrm{~K} 1 \mathrm{C}$ renovascular hypertension, ${ }^{35} 3$ weeks after clipping the blood pressure, PRA and urinary 8-isoprostane were all elevated. Acute tempol administration reduced blood pressure and increased renal perfusion suggesting a significant role for superoxide in regulating renal hemodynamics in this model of renal hypertension. About 20 weeks of $20 \%$ high fructose and $8 \%$ high sodium has been reported to elevate oxidative stress and induce hypertension. ${ }^{36}$ Cowley et $\mathrm{al}^{27}$ have elegantly reviewed how increased reactive oxygen species or reduced scavenging of reactive oxygen species results in reduced renal medullary blood flow and retarded sodium excretion resulting in hypertension. Nishimoto et $\mathrm{al}^{37}$ found that high dietary fructose diminished renal medullary endothelial nitric oxide synthase in salt-sensitive rats. While we do not have urinary $\mathrm{NO}_{2} / \mathrm{NO}_{3}$ measurements in the current data, the combination of elevated reactive oxygen species and our previously reported ${ }^{7}$ diminished NO bioavailability should lead to increased renal vascular resistance ${ }^{35}$ and decreased natriuresis, ${ }^{7,20}$ both of which could contribute to the hypertension. Tempol treatment should reverse this trend, and thereby explain the antihypertensive response we observed in our fructose plus HS rats.

Renin, the rate-limiting enzymatic step in the formation of Ang II, is inhibited by high renal perfusion pressure and by HS intake. ${ }^{8,9}$ The bioactive molecule of this system is Ang II, which has intrinsic vasoconstrictor properties, is a potent pressor factor ${ }^{8}$ and has been found to have a sensitive interaction with the vasodilator and natriuretic nitric oxide in the regulation of renal perfusion. ${ }^{38,39}$ Ang II is one of the most potent endogenous stimuli for oxygen-free radical formation. ${ }^{40-42}$ High fructose and HS intake has been shown to induce renin-angiotensin-stimulated renal reactive oxygen species. ${ }^{43}$ In addition, it has been reported that reactive oxygen species can stimulate the secretion of renin ${ }^{21}$ directly from renin-synthesizing juxtaglomerular cells. We have found previously ${ }^{7}$ as well as in the current study that $20 \%$ fructose intake reverses the inhibition of renin by HS and high renal perfusion pressure such that it remains inappropriately normal. We have also seen (unpublished observations) that after 4 weeks of HS, a further $80 \%$ suppression of renin, and this is also reversed and elevated by coincident salt plus $20 \%$ fructose intake. This increase is not seen with $20 \%$ fructose and normal salt. ${ }^{7}$ Several reports have found that pharmacologic suppression of the renin angiotensin system blunts or reverses fructose-induced hypertension, ${ }^{14,15,44}$ suggesting that the inappropriate maintenance of PRA may contribute to this hypertension. Note that our results are from only the first 2 weeks of $20 \%$ fructose administration and may be quite different by $8-12$ weeks of $20 \%$ fructose when the model is complicated by the development of metabolic syndrome. Thus, one might envision a vicious positive feedback cycle in which fructose-induced free radical formation directly stimulates renin secretion, elevates Ang II, which in turn increases blood pressure, and further stimulates reactive oxygen species formation. These all would diminish nitric oxide bioavailability and amplify the pro-hypertensive factors, all of which are consistent with our current and previous results. ${ }^{6,7}$ Scavenging reactive oxygen species with tempol should reverse all of this, and we found that it did reverse the hypertension and restored the appropriate renin response to HS intake. We did not see any effect of tempol treatment on PRA in the HS + T group. Since HS had already suppressed PRA, it would be difficult to further determine whether tempol exerted any additional effect in this group.

\section{Conclusion}

We hypothesized that at least part of the pathway leading to fructose-induced salt sensitive hypertension could be due to fructose-induced reactive oxygen species formation and inappropriate stimulation of renin secretion that would be reversed by the SOD mimetic tempol. We found that tempol treatment completely reversed the fructose-induced salt-sensitive hypertension, diminished the excretion of 8-isoprostane, and restored the appropriate HS suppression of renin secretion. Thus, we conclude that much of the effect of fructose-induced salt-sensitive hypertension is driven by renal formation of reactive oxygen species.

\section{Acknowledgment}

This research was supported by funding from the National Institutes of Health Grant 5P01HL090550-05 and funds from Henry Ford Hospital.

\section{Disclosure}

The authors report no conflicts of interest in this work. 


\section{References}

1. Johnson RJ, Segal MS, Sautin Y, et al. Potential role of sugar (fructose) in the epidemic of hypertension, obesity and the metabolic syndrome, diabetes, kidney disease and cardiovascular disease. Am J Clin Nutr. 2007;86(4):899-906.

2. Yang Q, Zhang Z, Gregg EW, et al. Added sugar intake and cardiovascular diseases mortality among US adults. JAMA Intern Med. 2014;174:516-524.

3. Hwang IS, Ho H, Hoffman BB, Reaven GM. Fructose-induced insulin resistance and hypertension in rats. Hypertension. 1987;10(5):512-516.

4. Mamikutty N, Thent ZC, Sapri SR, Sahruddin NN, Mohd Yusof MR, Haji Suhaimi F. The establishment of metabolic syndrome model by induction of fructose drinking water in male Wistar rats. Biomed Res Int. 2014;2014:1-8.

5. Shultz PJ, Tolins JP. Adaptation to increased dietary salt intake in the rat. Role of endogenous nitric oxide. J Clin Invest. 1993;91(2):642-650.

6. Cabral PD, Hong NJ, Hye Khan MA, et al. Fructose stimulates $\mathrm{Na} / \mathrm{H}$ exchange activity and sensitizes the proximal tubule to angiotensin II. Hypertension. 2014;63(3):e68-e73.

7. Gordish KL, Kassem KM, Ortiz PA, Beierwaltes WH. Moderate (20\%) fructose-enriched diet stimulates salt-sensitive hypertension with increased salt retention and decreased renal nitric oxide. Physiol Rep. 2017;5(7):E13162-E13178.

8. Beierwaltes WH. The role of calcium in the regulation of renin secretion. Am J Physiol Renal Physiol. 2010;298(1):F1-F11.

9. Castrop H, Höcherl K, Kurtz A, et al. Physiology of kidney renin. Physiol Rev. 2010;90:607-673.

10. Franco M, Tapia E, Santamaría J, et al. Renal cortical vasoconstriction contributes to development of salt-sensitive hypertension after angiotensin II exposure. J Am Soc Nephrol. 2001;12(11):2263-2271.

11. Guzik TJ, Hoch NE, Brown KA, et al. Role of the T cell in the genesis of angiotensin II induced hypertension and vascular dysfunction. $J$ Exp Med. 2007;204(10):2449-2460.

12. Johnson MD, Zhang HY, Kotchen TA. Sucrose does not raise blood pressure in rats maintained on a low salt intake. Hypertension. 1993; 21(6 pt 1):779-785.

13. Nakayama T, Kosugi T, Gersch M, et al. Dietary fructose causes tubulointerstitial injury in the normal rat kidney. Am J Physiol Renal Physiol. 2010;298:F710-F712.

14. Chou CL, Lai YH, Lin TY, Lee TJ, Fang TC. Aliskiren prevents and ameliorates metabolic syndrome in fructose-fed rats. Arch Med Sci. 2011;7:882-888.

15. Chou CL, Pang CY, Lee TJ, Fang TC. Direct renin inhibitor prevents and ameliorates insulin resistance, aortic endothelial dysfunction and vascular remodeling in fructose-fed hypertensive rats. Hypertens Res. 2013;36(2):123-128.

16. Queiroz-Leite GD, Crajoinas RO, Neri EA, et al. Fructose acutely stimulates NHE3 activity in kidney proximal tubule. Kidney Blood Press Res. 2012;36(1):320-334.

17. Vokurková M, Rauchová H, Řezáčová L, Vaněčková I, Zicha J. ROS production is increased in the kidney but not in the brain of Dahl rats with salt hypertension elicited in adulthood. Physiol Res. 2015;64(3):303-312.

18. Dikalov SI, Nazarewicz RR. Angiotensin II-induced production of mitochondrial reactive oxygen species: potential mechanisms and relevance for cardiovascular disease. Antioxid Redox Signal. 2013;19(10):1085-1094.

19. Cabral PD, Garvin JL. Luminal flow regulates NO and O2(-) along the nephron. Am J Physiol Renal Physiol. 2011;300(5):F1047-F1053.

20. Gordish KL, Beierwaltes WH. Resveratrol induces acute endotheliumdependent renal vasodilation mediated through nitric oxide and reactive oxygen species scavenging. Am J Physiol Renal Physiol. 2014;306(5):F542-F550.

21. Galle J, Herzog C, Schollmeyer P, Wanner C. Oxygen-derived radicals stimulate renin release of isolated juxtaglomerular cells. FEBS Lett. 1994;351(3):314-316.
22. Jaiswal N, Maurya CK, Arha D, et al. Fructose induces mitochondrial dysfunction and triggers apoptosis in skeletal muscle cells by provoking oxidative stress. Apoptosis. 2015;20(7):930-947.

23. Fernandez JM, Da Silva-Grigoletto ME, Gomez-Puerto JR, et al. A dose of fructose induces oxidative stress during endurance strength exercise. J Sports Sci. 2009;27(12):1323-1334.

24. Badr KF, Abi-Antoun TE. Isoprostanes and the kidney. Antioxid Redox Signal. 2005;7(1-2):236-243.

25. Pettinger WA. Anesthesia and the renin-angiotensin-aldosterone axis. Anesthesiology. 1978;48:393-396.

26. Macgriff S, Woo RE, Ortiz-Capisano MC, Atchison DK, Beierwaltes WH. Recruited renin-containing renal microvascular cells demonstrate the calcium paradox regulatory phenotype. Integr Blood Press Control. 2014;7:9-17.

27. Cowley AW, Abe M, Mori T, et al. Reactive oxygen species as important determinants of medullary flow, sodium excretion and hypertension. Am J Physiol Renal Physiol. 2015;308:F179-F197.

28. Wilcox CS. Oxidative stress and nitric oxide deficiency in the kidney: a critical link to hypertension? Am J Physiol Regul Integr Comp Physiol. 2005;289(4):R913-R935.

29. Feng W, Dell'Italia LJ, Sanders PW. Novel paradigms of salt and hypertension. J Am Soc Nephrol. 2017;28(5):1362-1369.

30. Nguyen Dinh Cat A, Montezano AC, Burger D, Touyz RM. Angiotensin II, NADPH oxidase, and redox signaling in the vasculature. Antioxid Redox Signal. 2013;19(10):1110-1120.

31. Porto ML, Lírio LM, Dias AT, et al. Increased oxidative stress and apoptosis in peripheral blood mononuclear cells of fructose-fed rats. Toxicol In Vitro. 2015;29(8):1977-1981.

32. Khattab MM. Tempol, a membrane-permeable radical scavenger, attenuates peroxynitrite- and superoxide anion-enhanced carrageenan-induced paw edema and hyperalgesia: a key role for superoxide anion. Eur $J$ Pharmacol. 2006;548(1-3):167-173.

33. Yanes L, Romero D, Iliescu R, et al. Systemic arterial pressure response to two weeks of Tempol therapy in SHR: involvement of NO, the RAS, and oxidative stress. Am J Physiol Regul Integr Comp Physiol. 2005;288(4):R903-R908.

34. Wilcox CS. Effects of tempol and redox-cycling nitroxides in models of oxidative stress. Pharmacol Ther. 2010;126(2):119-145.

35. Guron GS, Grimberg ES, Basu S, Herlitz H. Acute effects of the superoxide dismutase mimetic tempol on split kidney function in two-kidney one-clip hypertensive rats. J Hypertens. 2006;24:387-394.

36. Dornas WC, Cardoso LM, Silva M, et al. Oxidative stress causes hypertension and activation of nuclear factor- $\kappa \mathrm{B}$ after high-fructose and salt treatments. Sci Rep. 2017;7:1-11.

37. Nishimoto Y, Tomida T, Matsui H, Ito T, Okumura K. Decrease in renal medullary endothelial nitric oxide synthase of fructose-fed, salt-sensitive hypertensive rats. Hypertension. 2002;40(2):190-194.

38. Sigmon DH, Newman JM, Beierwaltes WH. Angiotensin II: endothelium-derived nitric oxide interaction in conscious rats. J Am Soc Nephrol. 1994;4(9):1675-1682.

39. Sigmon DH, Beierwaltes WH. Angiotensin II: nitric oxide interaction and the distribution of blood flow. Am J Physiol. 1993;265(6 pt 2):R1276-R1283.

40. Hitomi H, Kiyomoto H, Nishiyama A. Angiotensin II and oxidative stress. Curr Opin Cardiol. 2007;22(4):311-315.

41. Hussain MB, Püntmann VO, Mayr M, Khong T, Singer DR. The role of oxidant stress in angiotensin II-mediated contraction of human resistance arteries in the state of health and the presence of cardiovascular disease. Vascul Pharmacol. 2006;45(6):395-399.

42. Massey KJ, Hong NJ, Garvin JL. Angiotensin II stimulates superoxide production in the thick ascending limb by activating NOX4. Am J Physiol Cell Physiol. 2012;303(7):C781-C789.

43. Su Q, Huo CJ, Li HB, et al. Renin-angiotensin system acting on reactive oxygen species in paraventricular nucleus induces sympathetic activation via AT1R/PKC $\gamma /$ Rac1 pathway in salt-induced hypertension. Sci Rep. 2017;7:1-13

44. Farah V, Elased KM, Morris M. Genetic and dietary interactions; role of angiotensin AT1a receptors in response to a high fructose diet. Am J Physiol Heart Circ Physiol. 2007;293:H1083-H1089. 
Integrated Blood Pressure Control is an international, peer-reviewed open-access journal focusing on the integrated approach to managing hypertension and risk reduction. Treating the patient and comorbidities together with diet and lifestyle modification and optimizing healthcare resources through a multidisciplinary team approach constitute key
Dovepress

features of the journal. This journal is indexed on American Chemical Society's Chemical Abstracts Service (CAS). The manuscript management system is completely online and includes a very quick and fair peerreview system, which is all easy to use. Visit http://www.dovepress.com/ testimonials.php to read real quotes from published authors.

Submit your manuscript here: https://www.dovepress.com/integrated-blood-pressure-control-journal 